\title{
The pseudokinase tribbles homolog 3 interacts with ATF4 to negatively regulate insulin exocytosis in human and mouse $\beta$ cells
}

\author{
Chong Wee Liew, ${ }^{1}$ Jacek Bochenski, ${ }^{2}$ Dan Kawamori, ${ }^{1}$ Jiang Hu, ${ }^{1}$ Colin A. Leech, ${ }^{3}$ \\ Krzysztof Wanic, ${ }^{2,4}$ Maciej Malecki, ${ }^{4}$ James $\mathrm{H}$. Warram, ${ }^{2}$ Ling Qi, $, 5,6$ \\ Andrzej S. Krolewski, ${ }^{2}$ and Rohit N. Kulkarni ${ }^{1}$

\begin{abstract}
${ }^{1}$ Section of Islet Cell and Regenerative Medicine, ${ }^{2}$ Section of Genetics and Epidemiology, Research Division, Joslin Diabetes Center and Department of Medicine, Harvard Medical School, Boston, Massachusetts, USA. ${ }^{3}$ Department of Medicine, State University of New York Upstate Medical University, Syracuse, New York, USA. ${ }^{4}$ Department of Metabolic Disorders, School of Medicine, Jagellonian University, Krakow, Poland. 5Division of Nutritional Sciences, Cornell University, Ithaca, New York, USA. ${ }^{6}$ Laboratories for Peptide Biology, Salk Institute for Biological Studies, La Jolla, California, USA.
\end{abstract}

\begin{abstract}
Insufficient insulin secretion and reduced pancreatic $\beta$ cell mass are hallmarks of type 2 diabetes (T2DM). Here, we confirm that a previously identified polymorphism (rs2295490/Q84R) in exon 2 of the pseudokinaseencoding gene tribbles 3 (TRB3) is associated with an increased risk for T2DM in 2 populations of people of mixed European descent. Carriers of the $84 \mathrm{R}$ allele had substantially reduced plasma levels of C-peptide, the product of proinsulin processing to insulin, suggesting a role for TRB 3 in $\beta$ cell function. Overexpression of TRB3 84R in mouse $\beta$ cells, human islet cells, and the murine $\beta$ cell line MIN6 revealed reduced insulin exocytosis, associated with a marked reduction in docked insulin granules visualized by electron microscopy. Conversely, knockdown of TRB3 in MIN6 cells restored insulin secretion and expression of exocytosis genes. Further analysis in MIN6 cells demonstrated that TRB3 interacted with the transcription factor ATF4 and that this complex acted as a competitive inhibitor of cAMP response element-binding (CREB) transcription factor in the regulation of key exocytosis genes. In addition, the 84R TRB3 variant exhibited greater protein stability than wild-type TRB3 and increased binding affinity to Akt. Mice overexpressing TRB3 84R in $\beta$ cells displayed decreased $\beta$ cell mass, associated with reduced proliferation and enhanced apoptosis rates. These data link a missense polymorphism in human TRB3 to impaired insulin exocytosis and thus increased risk for T2DM.
\end{abstract}

\section{Introduction}

Diabetes mellitus, primarily characterized by chronic hyperglycemia, is one of the most serious metabolic disorders and is projected to affect 366 million people worldwide by 2030 (1). While insulin resistance plays a causal role in the pathogenesis and development of type 2 diabetes (T2DM) and its complications $(2,3)$, there is general consensus that an inability of $\beta$ cells to compensate for peripheral insulin resistance triggers the development of overt diabetes (4).

The insulin/Akt signaling pathway regulates mitogenic effects in virtually all metabolic tissues, including pancreatic $\beta$ cells (5), and proteins that inhibit Akt activity are considered potential inducers of insulin resistance (6). An example of such a protein is a catalytically inactive pseudokinase termed tribbles homolog 3 (TRB3) (7). Recently, a polymorphism (rs2295490/Q84R) was identified in exon 2 of $T R B 3$, in which a base-pair substitution resulted in a change from glutamine to arginine at position 84 of the protein (8). Individual carriers for the arginine variant (84R) exhibit higher risk for T2DM, implicating a pathological role for the Q84R polymorphism (8).

In the present study, we confirmed the association of the Q84R polymorphism with T2DM in cohorts of people of mixed European descent from Boston, Massachusetts, USA, and Krakow, Poland. Our preliminary findings of reduced C-peptide levels in carriers of

Conflict of interest: The authors have declared that no conflict of interest exists. Citation for this article: J Clin Invest. 2010;120(8):2876-2888. doi:10.1172/JCI36849. the polymorphism, and a recent paper by Prudente et al. (9) reporting insulin secretory defects in islets isolated from donors from an independent cohort of carriers of the polymorphism, prompted us to focus our efforts on understanding the mechanisms underlying the role of TRB3 in $\beta$ cell biology.

In this manuscript, we specifically explored the functional effects of expressing the $84 \mathrm{R}$ variant in pancreatic $\beta$ cells in mice and expression of the 84R or Q84 variants in human islet cells and mouse $\beta$ cell lines. We observed a significant inhibition of insulin secretion and proliferation coupled with enhanced apoptosis in $\beta$ cells expressing the TRB3 variants. In addition, we report that TRB3 inhibits insulin secretion by downregulating specific proteins in the exocytosis machinery through competitive inhibition of cAMP response element-binding (CREB) transcriptional activity, an upstream activating transcription factor of the exocytosis genes. Together, these data implicate TRB3 as a potential target to improve $\beta$ cell function for the treatment of T2DM.

\section{Results}

TRB3 84 variant is associated with risk of T2DM. In cohorts of people of mixed European descent from Boston, Massachusetts, USA, and Krakow, Poland, we genotyped 702 nondiabetic subjects and 766 T2DM subjects and observed an association of the Q84R TRB3 polymorphism with T2DM (see Supplemental Methods for genetic studies; supplemental material available online with 
A
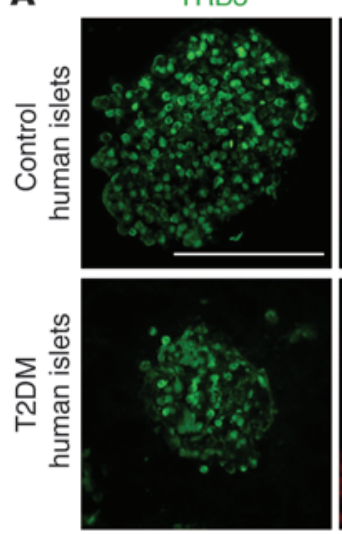

B

FACS-sorted mouse islet cells Trb3 mRNA
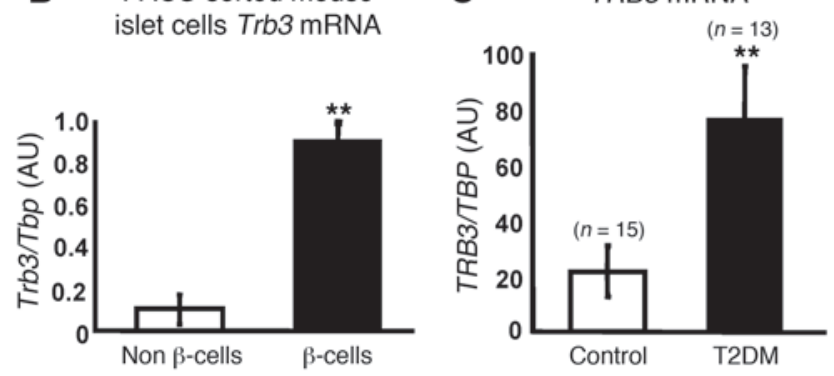

Insulin
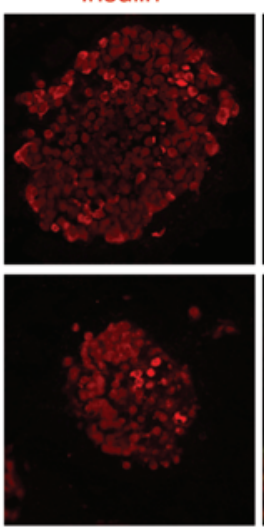

Merge
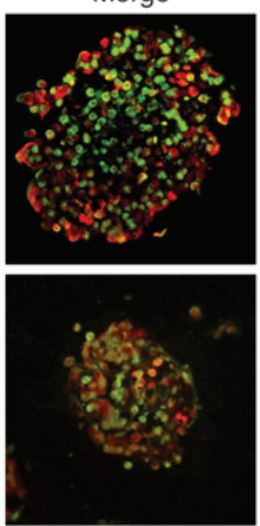

TRB3
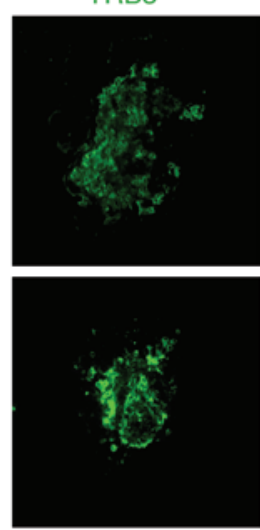

Glucagon
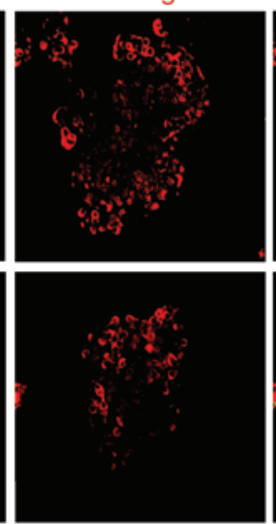

Merge
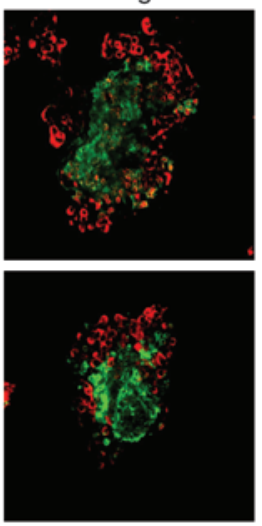

D

\section{TRB3}

Tubulin

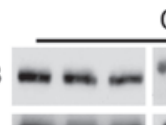

Control

$=\mathrm{n}$
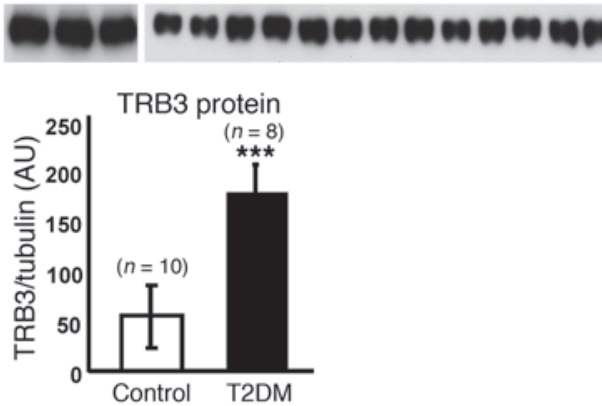
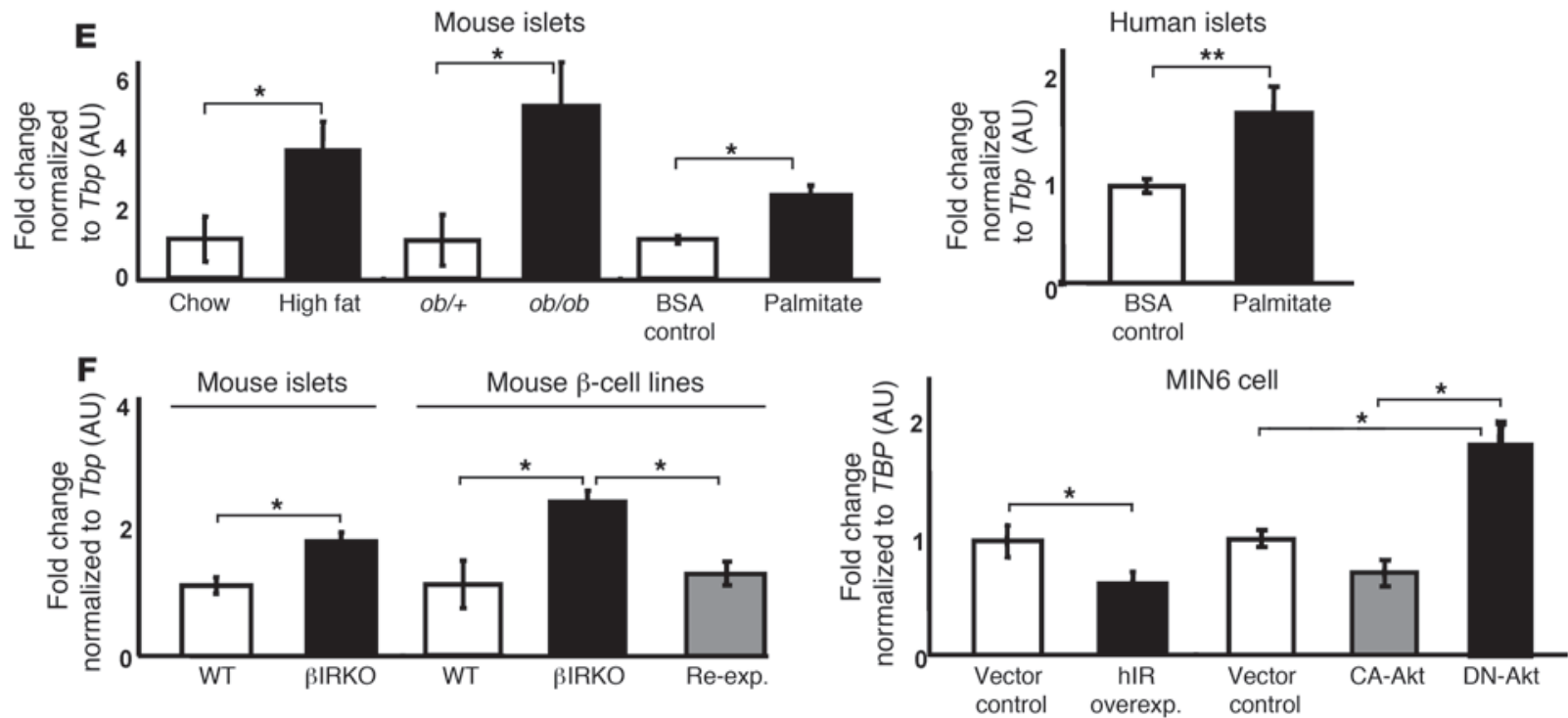

Figure 1

Regulation of TRB3 expression in human and mouse islets. (A) Immunofluorescence staining of agarose-embedded human control and T2DM islets for TRB3 (green), insulin (red), and glucagon (red). Scale bar: $50 \mu \mathrm{m}$. Original magnification, $\times 40$. (B and C) qPCR for Trb3 mRNA in (B) FACS-sorted mouse islet cells ( $n=3$ in each group) and (C) human control and T2DM islets $(n=13-15)$. TBP, TATA box binding protein. (D) Western blotting for TRB3 in human control and T2DM islets $(n=8-10)$. White vertical lines indicate noncontiguous lanes. (E) qPCR for Trb3 mRNA in mouse islets from high-fat diet-fed (high fat) or chow-fed (chow) mice ( $n=4$ in each group), ob/ob or ob/+ control mice ( $n=4$ in each group), and mice treated with palmitate or BSA control ( $n=4$ in each group) or human islets ( $n=3$ in each group) treated with palmitate or BSA control. (F) qPCR for Trb3 mRNA in mouse islets from $\beta$ IRKO or control mice ( $n=4$ in each group), $\beta$ IRKO cell, insulin receptor reexpression (Re-Exp) cell, or control mouse cell lines ( $n=4$ in each group), and MIN6 cells infected with virus expressing human insulin receptor (hIR), CA-Akt, or DN-Akt ( $n=4$ in each group). Overexp., overexpressing. All data are presented as mean \pm SEM. ${ }^{\star} P<0.05,{ }^{* \star} P<0.01,{ }^{* \star *} P<0.001$. 
this article; doi:10.1172/JCI36849DS1). The R variant was associated with T2DM under all 3 genetic models, with the effect under the dominant model slightly stronger than under other models $(\mathrm{OR}=1.4$; with $95 \% \mathrm{CI}, 1.1-1.8$; and $P=0.012$ corrected for number of genetic models examined) (Supplemental Table 1). Our finding is consistent with recently published data by Prudente et al. (9). Since we did not find any other sequence difference that was in linkage disequilibrium with the Q84R polymorphism, this polymorphism can be considered as causally related to T2DM. Although the polymorphism can potentially impact the function of the liver, the significantly lower plasma C-peptide levels in the 84RR homozygotes compared with those in the QQ84 homozygotes (mean $\pm \mathrm{SD}, 1.88 \pm 0.63$ vs. $2.77 \pm 2.2 \mathrm{ng} / \mathrm{ml}, P=0.003$ ) suggested that the Q84R polymorphism has a pathogenic role in pancreatic $\beta$ cell failure.

TRB3 expression is elevated in islets from patients with T2DM and highfat fed-and insulin receptor-deficient mice. Since the expression of TRB3 in either rodent or human pancreas has, to our knowledge, not been reported, we systematically examined human islets and mouse islets isolated from different models of diabetes and obesity. Coimmunostaining of freshly embedded normal control and T2DM human islets revealed that TRB3 was localized to insulinpositive $\beta$ cells but not glucagon-positive $\alpha$ cells in both groups (Figure 1A). Further, quantitative real-time PCR (qPCR) of islet cells subjected to FACS confirmed that Trb3 was predominantly expressed in mouse $\beta$ cell fractions (Figure 1B and Supplemental Figure 1). Interestingly, qPCR analysis of islets from patients with T2DM revealed an approximately 4-fold increase in TRB3 expression and was consistent with an approximately 3 -fold increase in protein levels (Figure 1, C and D). We also observed an approximately 4-fold increase in Trb3 expression in islets isolated from high-fat diet-fed or $o b / o b$ mice (Figure 1E), indicating a role for TRB3 in obesity. Treating human and mouse islets with palmitate ( $0.4 \mathrm{M}, 48$ hours) resulted in a 2 -fold increase in TRB3 expression, linking the effects of fatty acids with TRB3 (Figure 1E). Next, we observed a 2-fold increase in Trb3 expression in islets or $\beta$ cell lines derived from $\beta$ cell insulin receptor knockout ( $\beta$ IRKO) mice (10) (Figure 1F, left panel). The increase in Trb3 levels was reversed by reexpression of the human insulin receptor in the $\beta$ IRKO mice (Figure 1F, right panel), while reduced Trb3 expression was evident in MIN6 cells overexpressing human insulin receptors (Figure 1F). Finally, inhibition of insulin signaling by dominant-negative Akt (DN-Akt) in MIN6 cells upregulated Trb3 expression, while the effects of constitutively active Akt (CA-Akt) showed a trend toward a decrease (Figure $1 \mathrm{~F}$ ). Together, these results clearly indicate that insulin signaling modulates Trb3 expression in pancreatic $\beta$ cells and suggest that inhibition of insulin signaling, which potentially occurs in $\beta$ cells during long-standing obesity and/or T2DM, leads to upregulation of TRB3 expression.

TRB3 inhibits glucose-stimulated insulin secretion and reduces $\beta$ cell mass in vivo. To directly investigate the role of TRB3 in vivo, we created a mouse model overexpressing TRB 3 in pancreatic $\beta$ cells using the rat insulin promoter (RIP) (Supplemental Figure 2A). A 4-fold increase of TRB3 in the islets of the RIP-TRB3 hemizygous mice (Figure 2A), which was similar to the increase in islets from T2DM humans (Figure 1, C and D), provided us with a suitable model for further studies. The presence of glucose intolerance in 2 independent founder lines (RIP-TRB3F1 and RIP-TRB3F2) excluded the possibility that the defects occurred due to an insertional artifact secondary to a transgene position effect (Supple- mental Figure 2B). For all subsequent studies, we focused on one of the founders (RIP-TRB3F1). RIP-TRB3F1 mice did not exhibit alterations in body weight or insulin sensitivity (Supplemental Figure 2, C and D). However, the mutants exhibited an age-dependent worsening of glucose tolerance (Figure $2 \mathrm{~B}$ ) that was, in part, due to defects in in vivo glucose-stimulated insulin secretion (GSIS) (Figure $2 \mathrm{C}$ ) and/or to significantly reduced $\beta$ cell mass (Figure 2D) (11). The insulin content, normalized to DNA, was similar between groups (data not shown). The presence of significantly fewer $\mathrm{BrdU}^{+} \beta$ cells indicated a defect in proliferation (Figure 2E), while the apoptosis rate, assessed by TUNEL staining, tended to be higher in the RIP-TRB3F1 mice (Figure 2F). These results suggest that TRB 3 overexpression impairs $\beta$ cell proliferation and promotes $\beta$ cell apoptosis in vivo, together leading to impaired insulin secretion and reduced $\beta$ cell mass.

TRB 3 inbibits $\beta$ cell growth and promotes apoptosis. To address the functional consequences of the TRB 3 variants in $\beta$ cells, we transduced human islets or MIN6 cells with recombinant adenovirus expressing either the Q84 or 84R variant and achieved approximately 4-fold higher levels of TRB3 (Figure 3F), a level that was comparable with that observed in human islets isolated from T2DM patients (Figure 1D). MIN6 cells expressing either variant showed significantly reduced growth, with the greatest deficit in $\beta$ cells expressing the $84 \mathrm{R}$ variant (Figure $3 \mathrm{~A}$ ). Significantly fewer number of $\mathrm{BrdU}^{+} \beta$ cells were observed in Q84 TRB3- or 84R TRB3-expressing $\beta$ cells (Figure $3 \mathrm{~B}$ ), consistent with our observation in RIP-TRB3F1 mice. A significantly greater percentage of TUNEL $\beta$ cells (Figure 3C) was consistent with the elevated levels of cleaved caspase 3 protein in MIN6 cells expressing 84R TRB3 (Figure 3D), suggesting that apoptosis contributed to the cell growth deficit (12). Furthermore, the expression of Bax, a proapoptotic protein that is regulated by Akt (13), was significantly increased in MIN6 cells expressing 84R TRB3 (Figure 3D). Similar results were confirmed in dispersed primary human islet cells (Figure $3 \mathrm{E}$ ). Together, these data suggest that TRB3 inhibits proliferation and enhances apoptosis in $\beta$ cells and the 84R TRB3 variant manifests a stronger phenotype among the 2 variants.

Q84 TRB3 or 84R TRB3 suppress Akt and ERK in dispersed human islet cells or MIN6 cells. TRB3 inhibits Akt (7) and MAPK pathways (14), both of which have been reported to regulate $\beta$ cell mass $(5,11)$. Expression of Q84 TRB3 or 84R TRB3 in human dispersed islet cells, significantly inhibited ERK phosphorylation in the basal state and phosphorylation of Akt and ERK following insulin stimulation (Figure 3F). The inhibitory effects on Akt activation were significantly greater in the 84R TRB3 group compared with those of the Q84 TRB3 group, while the ability to inhibit ERK activation after insulin stimulation was similar between groups (Figure 3F). Similar results were observed in MIN6 cells (Supplemental Figure 3, A and B). The lack of significant alterations in the level of LKB1 (15) and activation of AMPK $\alpha$ (16) or ACC (17) confirmed the specific effects of Q84 and 84R TRB3 on Akt and ERK in MIN6 cells (Supplemental Figure 4). The lack of differences in activation of PERK and expression of XBP1 and BiP between groups (data not shown) indicated that the alterations in insulin signaling are unlikely to be due to direct activation of ER stress responses (18).

TRB3 inhibits insulin secretion in response to glucose and non-glucose secretagogues. We next investigated the effects of TRB3 on insulin exocytosis. Dispersed human islet cells (Figure 4A and Supplemental Figure 5A) or MIN6 cells (Figure 4B and Supplemental Figure $5 B$ ) expressing either Q84 TRB3 or 84R TRB3 displayed a time- 
A

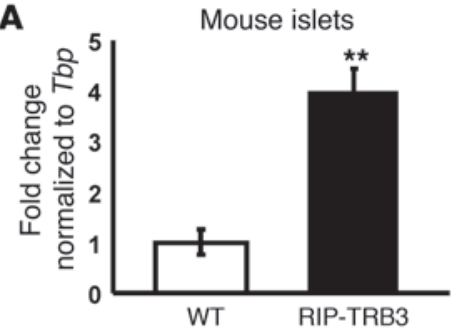

C

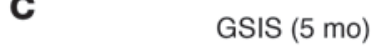

B

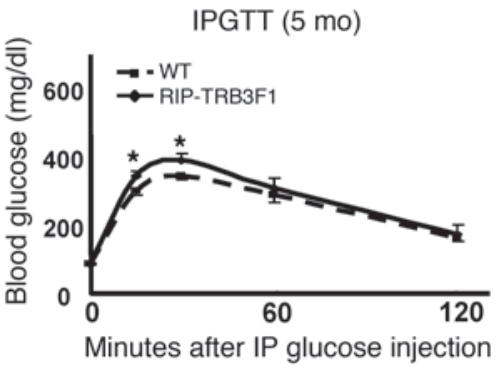

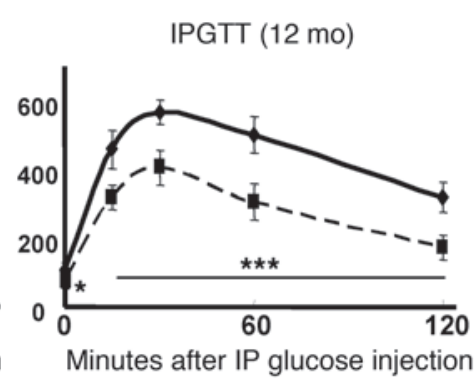

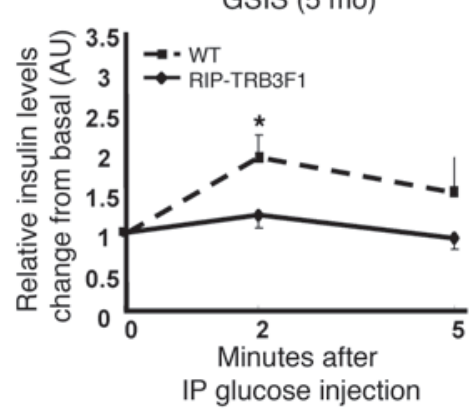

GSIS (12 mo)

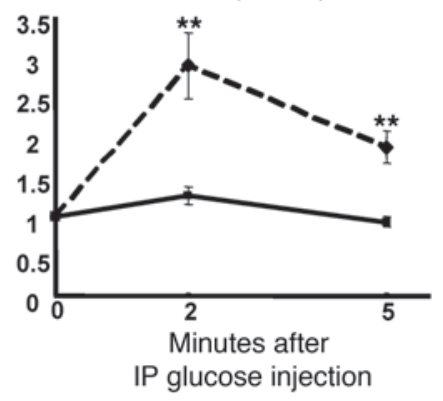

D Insulin/Glucagon

Insulin/Glucagon
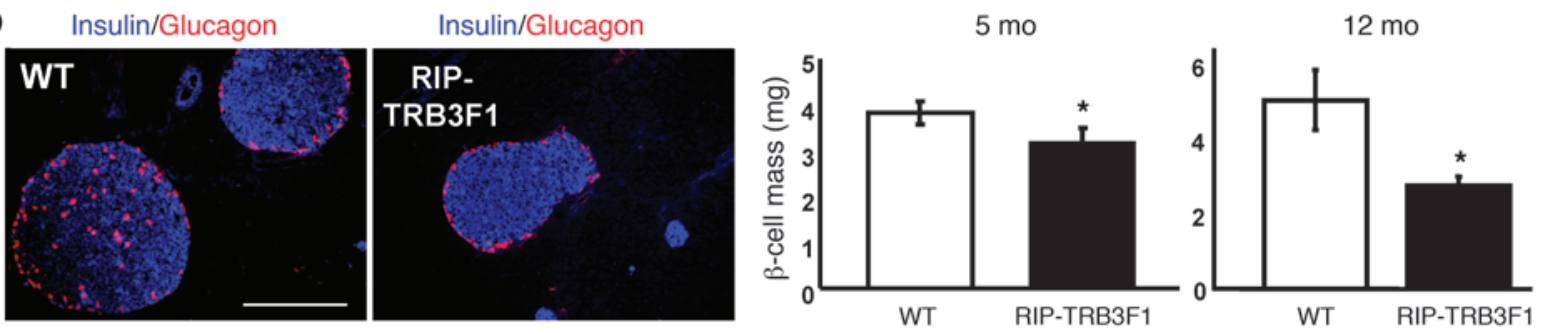

$\mathbf{E}$ $5 \mathrm{mo}$
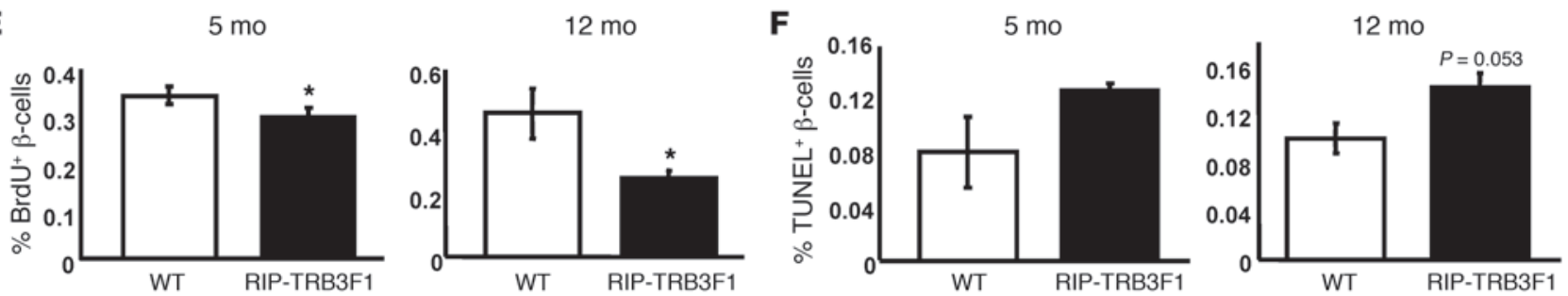

Figure 2

Impaired insulin secretion and reduced $\beta$ cell mass in RIP-TRB3F1 mice. (A) qPCR for Trb3 mRNA in mouse islets from RIP-TRB3F1 and control mice ( $n=4$ in each group). (B) Glucose tolerance in 5-month-old and 12-month-old male mice (glucose, $2 \mathrm{~g} / \mathrm{kg}$ body weight, $n=10-12$ ). IPGTT, intraperitoneal glucose tolerance test. (C) In vivo GSIS in 5-month-old and 12-month-old male mice (glucose, $3 \mathrm{~g} / \mathrm{kg}$ body weight, $n=8$ in each group). (D) Immunofluorescence staining of pancreas sections for insulin (blue) and glucagon (red) and $\beta$ cell mass ( $n=4$ in each group) in 5-month-old and 12-month-old male mice. Scale bar: $100 \mu \mathrm{m}$. (E) BrdU-positive $\beta$ cells and (F) TUNEL-positive $\beta$ cells in 5-month-old and 12-month-old male mice ( $n=4$ in each group). All data are presented as mean \pm SEM. ${ }^{\star} P<0.05,{ }^{\star \star} P<0.01,{ }^{\star \star \star} P<0.001$.

dependent reduction of insulin secretion at stimulatory glucose concentrations, with the 84R TRB3 variant consistently exhibiting a significantly greater inhibition. A similar secretory pattern was observed in response to L-arginine or $\mathrm{KCl}$ (Figure 4, C and D), and no significant differences in insulin content were observed among groups (data not shown). Consistent with these data, we observed a significant inhibition of insulin secretion in primary islets isolated from RIP-TRB3F1 mice (Figure 4E). Conversely, when TRB3 was knocked down in MIN6 cells expressing LacZ, Q84 TRB3, or $84 \mathrm{R}$ TRB3 or knocked down in cells treated with the ER stress inducer, thapsigargin (which increases TRB3 expression; ref. 19), we noted a significant improvement in insulin secretion (Figure 4F). Expression of TRB3 variants did not significantly affect cAMP production (Supplemental Figure 6A) or exhibit differences in glucose-stimulated $\left[\mathrm{Ca}^{+}\right]_{i}$ flux measurements (Supplemental Figure $6, \mathrm{~B}-\mathrm{F})$. These data indicate that the defect in insulin secretion in $\beta$ cells expressing TRB3 occurs distal to $\mathrm{Ca}^{2+}$ influx and likely at the level of insulin exocytosis.

TRB3 inhibits the expression of key proteins in the insulin exocytosis pathway. To localize the proteins mediating the inhibitory effects 
A

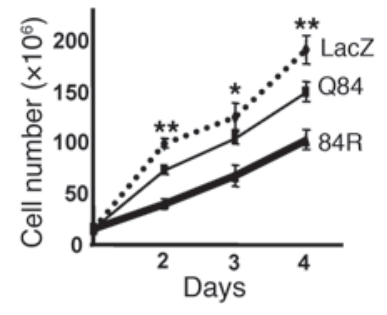

C

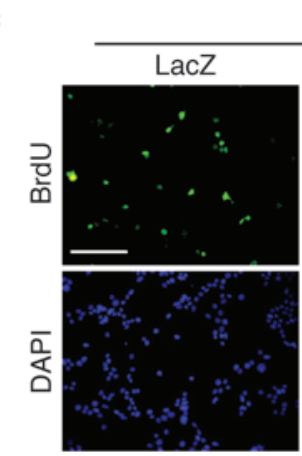

B

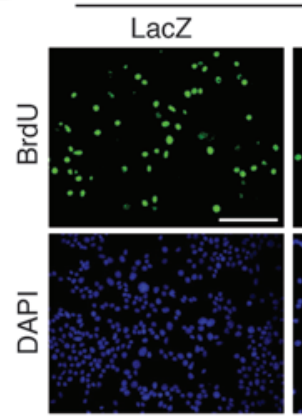

MIN6 cells

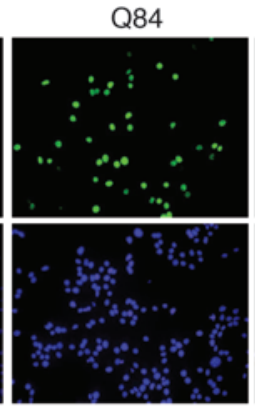

MIN6 cells
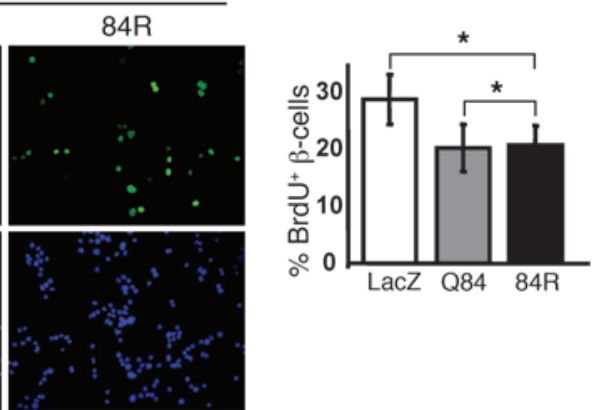

D
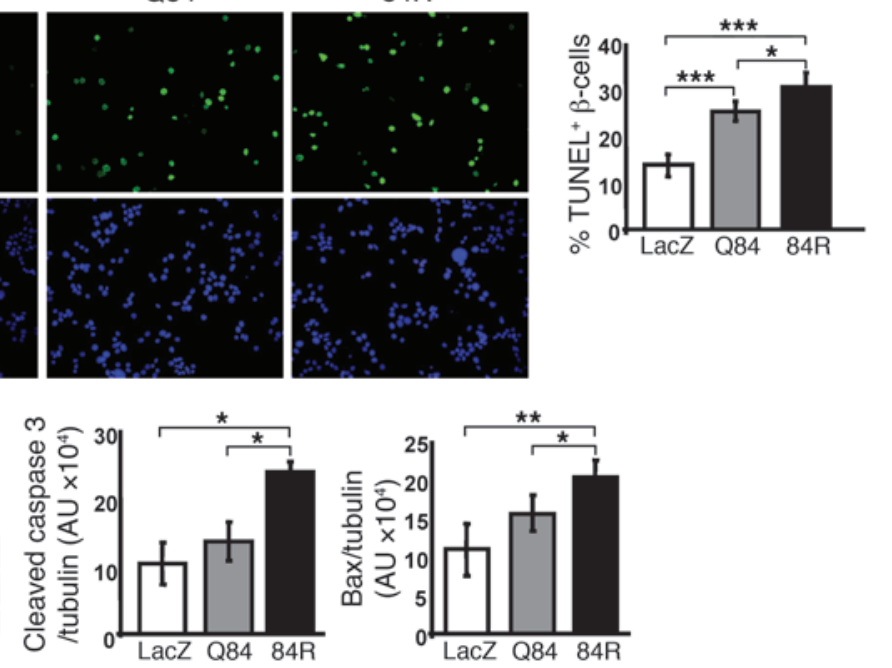

E

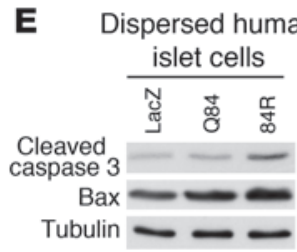

Tubulin
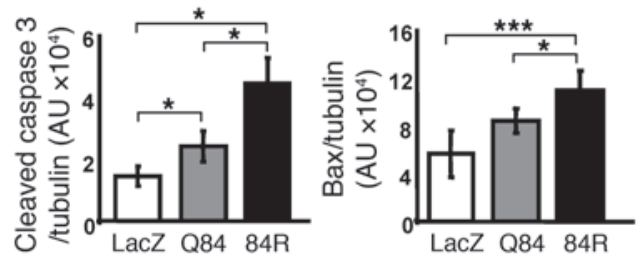

$\mathbf{F}$

Dispersed human islet cells

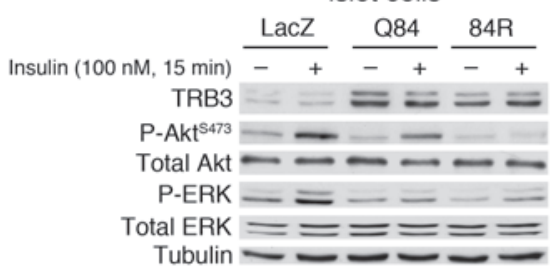

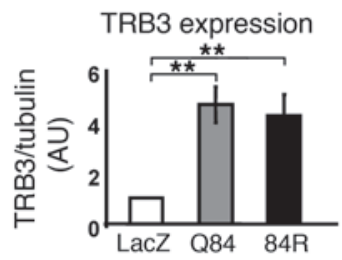

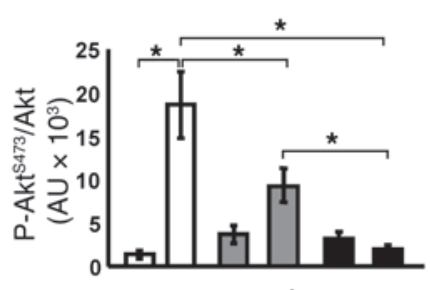

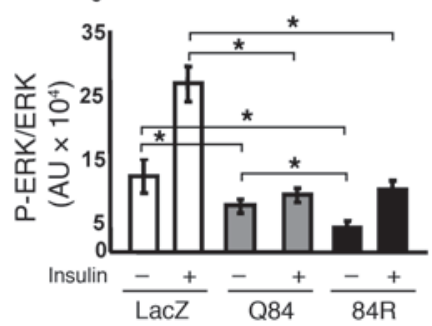

Figure 3

Reduced proliferation, increased apoptosis, and inhibition of insulin signaling in $\beta$ cells expressing Q84 TRB3 or 84R TRB3. (A) Number of MIN6 cells expressing LacZ, Q84 TRB3, or 84R TRB3 at the indicated time points ( $n=4$ in each group). (B) BrdU (green) incorporation into MIN6 cells expressing LacZ control, Q84 TRB3, or 84R TRB3 ( $n=4$ in each group). Scale bar: $50 \mu \mathrm{m}$. (C) TUNEL assay in MIN6 cells expressing LacZ, Q84 TRB3, or 84R TRB3 ( $n=4$ in each group). Scale bar: $50 \mu \mathrm{m}$. (D and $\mathbf{E})$ Western blotting for cleaved caspase 3 and Bax proteins in (D) MIN6 cells and (E) dispersed human islet cells expressing LacZ, Q84 TRB3, or 84R TRB3 ( $n=4$ in each group). (F) Western blotting for TRB3, total and phospho-Akt (Ser473), total and phosho-ERK in basal or insulin-stimulated dispersed human islet cells expressing LacZ, Q84 TRB3, or 84R TRB3. Data are expressed as AU of the Western blots and are depicted as a ratio of TRB3 to total Akt, phospho-Akt (Ser473) to total Akt, and p-ERK to total ERK ( $n=4$ in each group). All data are presented as mean \pm SEM. ${ }^{\star} P<0.05$, ${ }^{* \star} P<0.01,{ }^{* \star *} P<0.001$. 
A

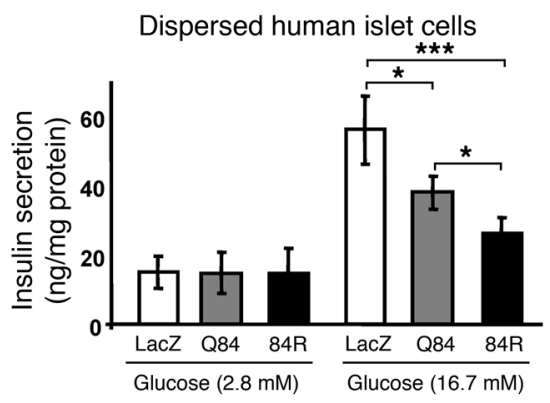

C

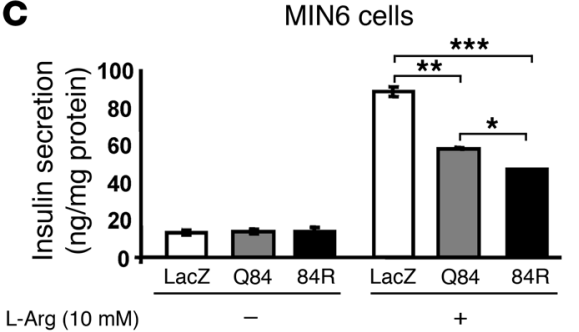

B

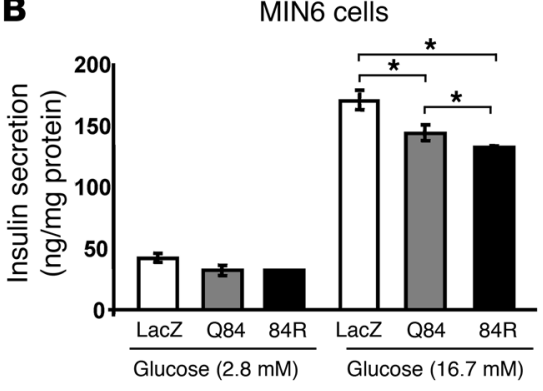

D

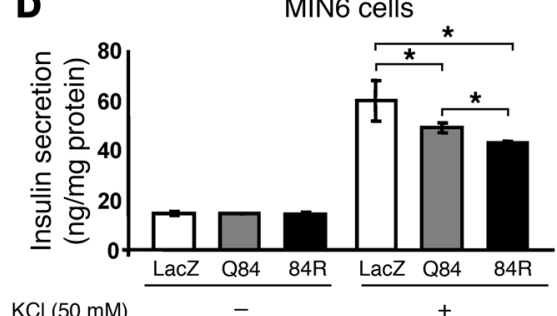

E

Isolated RIP-TRB3F1 mouse islets (6 mo)
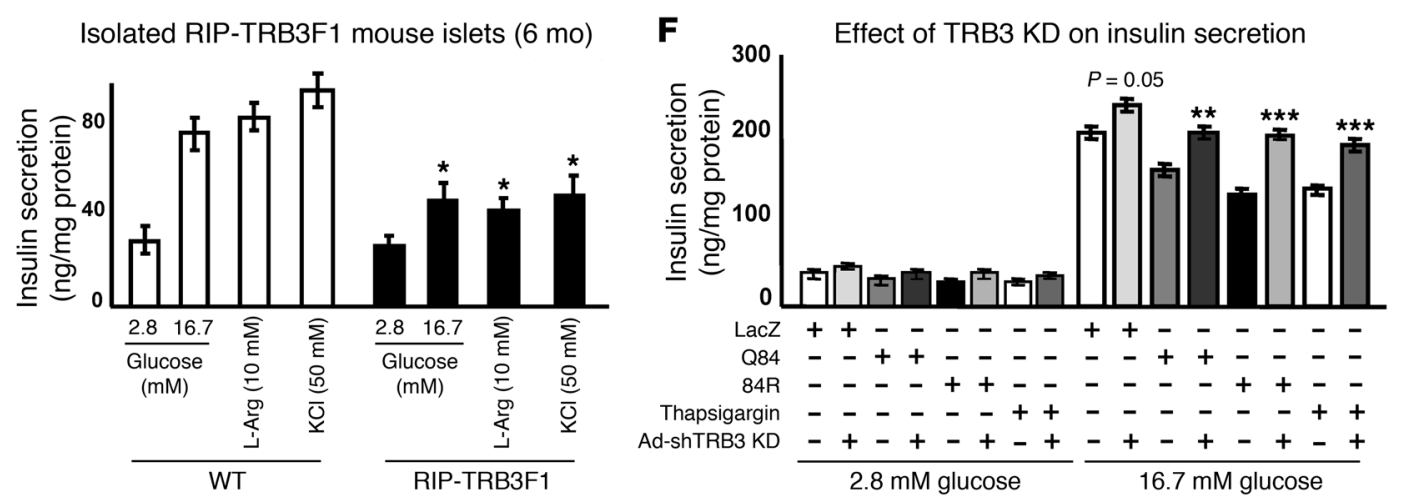

Figure 4

Reduced GSIS in human islet cells and MIN6 cells expressing Q84 TRB3 or 84R TRB3. (A and B) GSIS (60 minutes) in (A) dispersed human islet cells and (B) MIN6 cells expressing LacZ, Q84 TRB3, or 84R TRB3 ( $n=4$ in each group). (C) L-Arginine (10 mM) and (D) KCl (50 mM) stimulated insulin secretion (60 minutes) in MIN6 cells expressing LacZ, Q84 TRB3, or 84R TRB3 ( $n=3$ in each group). (E) Insulin secretion (60 minutes) in islets isolated from control and RIP-TRB3F1 mice ( $n=4$ in each group). (F) Insulin secretion (60 minutes) in MIN6 cells expressing LacZ, Q84 TRB3, or 84R TRB3 or in MIN6 cells treated with thapsigargin (100 nM), with or without Adenovirus-shTRB3 (Ad-shTRB3 KD) $(n=3$ in each group). All data are presented as mean \pm SEM. ${ }^{*} P<0.05,{ }^{* \star} P<0.01,{ }^{* \star} P<0.001$.

of TRB3 on insulin secretion, we analyzed the expression of key genes involved in glucose sensing, glucose metabolism, stimulus secretion coupling, insulin biosynthesis, and insulin signaling and did not observe differences among groups (Supplemental Figure 7, B-D). However, expression of either variant of TRB3 only resulted in a small but significant reduction in the transcription factor, Neurod1 (Figure 5A and Supplemental Figure 7A).

Gene and protein expression of the GTPases Rab3d and Rab27a, which participate in insulin exocytosis $(20,21)$, were decreased significantly in MIN6 cells expressing Q84 TRB3 or 84R TRB3, and the greatest reduction was evident with the 84R variant (Figure 5, $A$ and B), while expression of Rab3a, Rab3b, and Rab3c were unaffected (data not shown). Furthermore, the expression of soluble $\mathrm{NH}_{2}$-ethylmaleimide sensitive fusion protein attachment protein receptors (SNAREs), synaptosomal-associated protein 25 (Snap25) and vesicle-associated membrane protein 2 (Vamp2), which mediate the docking and fusion of secretory granules to the plasma membrane (21), was also decreased significantly in TRB3-expressing MIN6 cells (Figure 5, A and B). The expression of other SNARE proteins, including Syntaxin1a, Granuphilin-a, Munc-18a, Munc$18 \mathrm{~b}$, and Munc-18c, was unchanged (data not shown). Expression of the T2DM susceptibility gene calpain 10 (Capn10) (22) was also reduced in MIN6 cells expressing TRB3 (Figure 5A). Downregulation of Snap25, Rab3d, and Rab27a gene expression was reversed by knocking down TRB3 in MIN6 cells expressing LacZ, Q84 TRB3, or 84R TRB3 or in cells treated with the ER stress inducer, thapsigargin, which induces TRB3 expression (Figure 5C). Reconstitution of the downregulated proteins Snap25 and Rab3d, together but not individually, restored GSIS in both Q84 TRB3and 84R TRB3-expressing MIN6 cells (Figure 5D), confirming their role in TRB3-associated exocytosis. Supplemental Figure 8 shows baseline insulin secretion. The TRB3-independent role of Snap25 and Rab3d on insulin secretion was further confirmed by individual and double knockdown experiments. While individual 


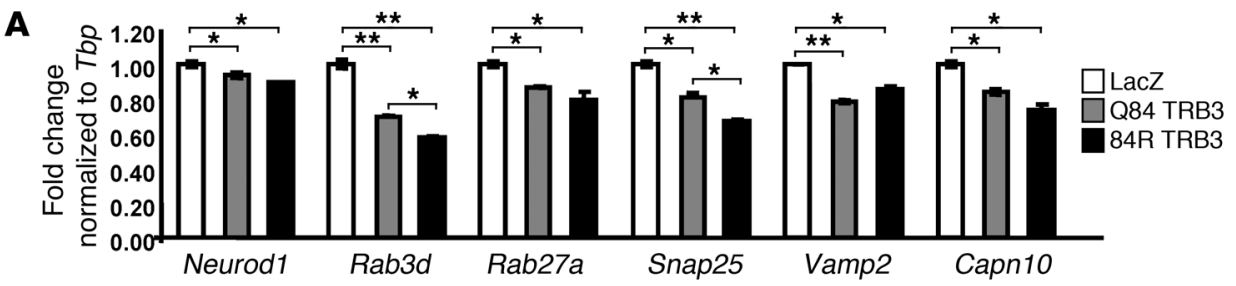

B
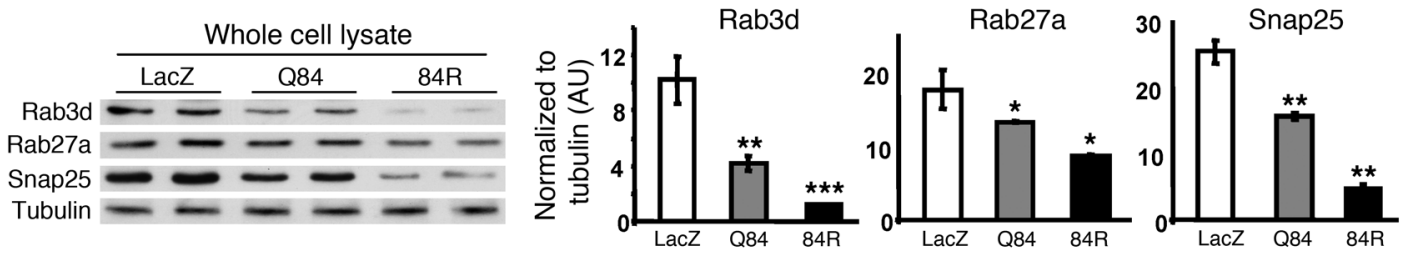

C

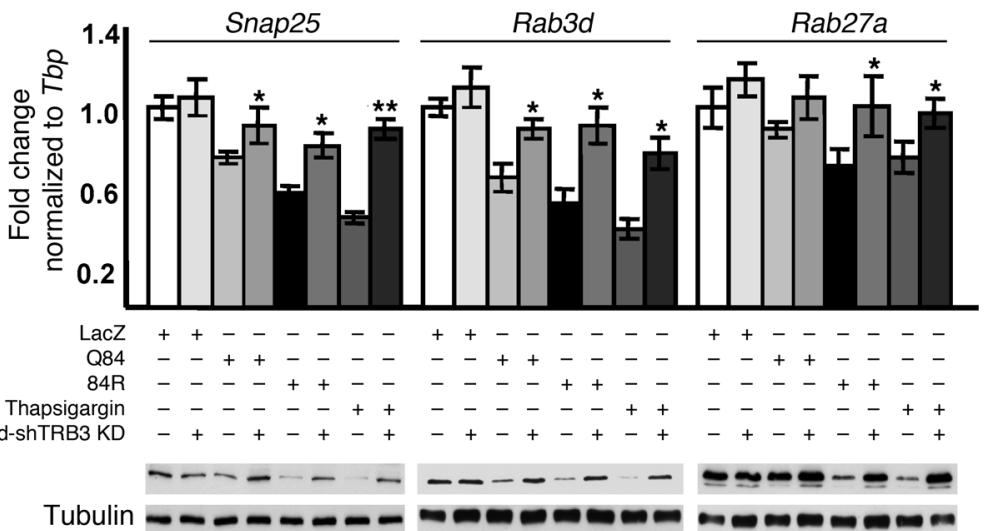

D

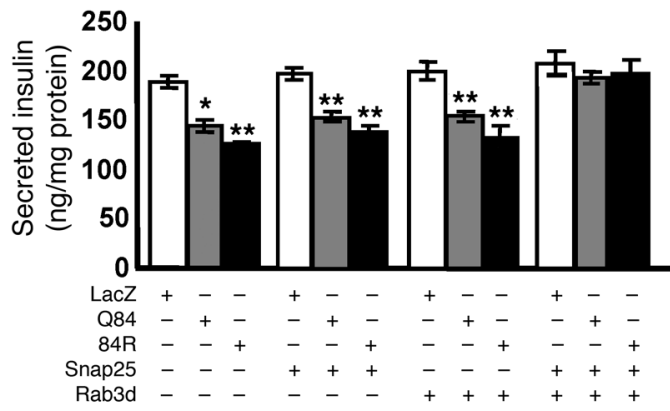

E

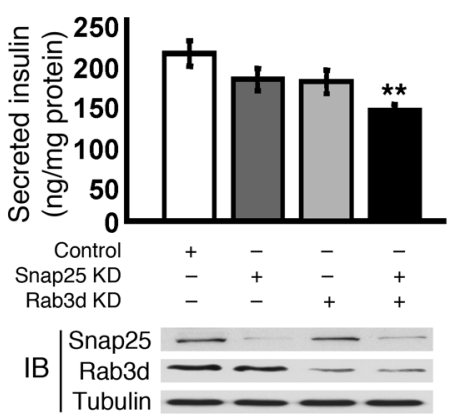

$\mathbf{F}$

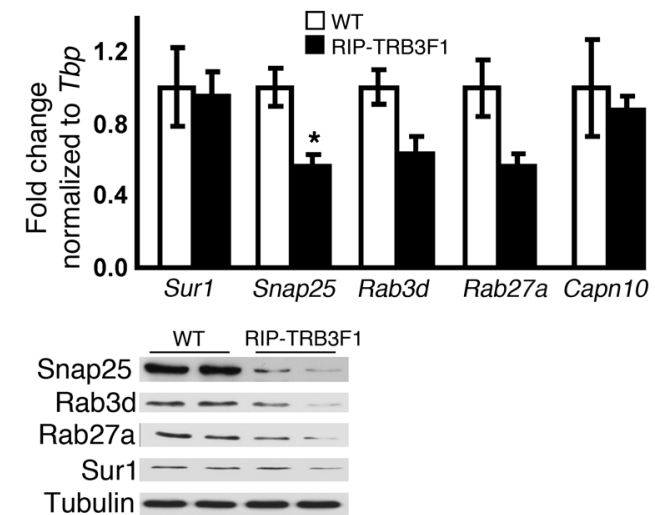

G

Control human islets $\quad$ T2DM human islets
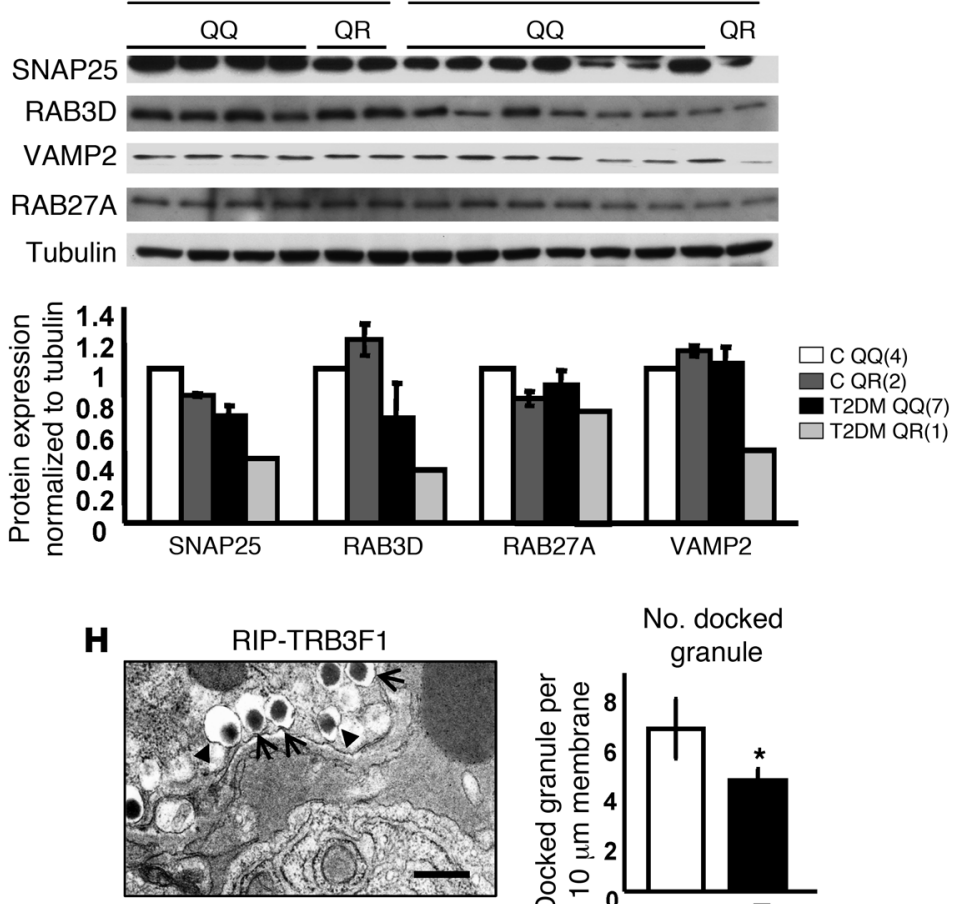

No. docked granule

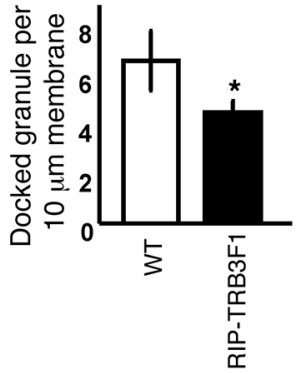




\section{Figure 5}

Reduced expression of genes/proteins involved in insulin exocytosis in MIN6 cells expressing Q84 TRB3 or 84R TRB3. (A) qPCR for insulin exocytosis genes in MIN6 cells expressing LacZ, Q84 TRB3, or 84R TRB3 ( $n=4$ in each group). (B) Western blotting for proteins involved in insulin secretion in MIN6 cells expressing LacZ, Q84 TRB3, or 84R TRB3 ( $n=3$ in each group). (C) qPCR and Western blotting for insulin exocytosis genes in MIN6 cells expressing LacZ, Q84 TRB3, or 84R TRB3 or MIN6 cells treated with thapsigargin (100 nM), with or without Adenovirus-shTRB3 ( $n=3$ in each group). For the top row of Western blots, each lane corresponds to the protein data for Snap25, Rab3d, and Rab27a shown in the graphs directly above. (D) Insulin secretion (60 minutes) in MIN6 cells expressing LacZ, Q84 TRB3, or 84R TRB3, with Snap25, Rab3d, both Snap25 and Rab3d, or alone ( $n=3$ in each group). (E) Insulin secretion (60 minutes) in MIN6 cells with control or with Snap25 or Rab3d knockdown (KD), alone or in combination ( $n=4$ in each group). (F) qPCR and Western blotting for insulin exocytosis genes in isolated islets from control or RIP-TRB3F1 mice $(n=4$ in each group). (G) Western blotting for SNAP25, RAB3D, RAB27A, and VAMP2 in human control and T2DM islets $(n=6-8)$. The numbers in the parentheses indicate the number of patients analyzed. C, Control. (H) Representative electromicroscopic image of docked (arrows) and undocked granules (arrowheads), and quantification of number of docked granules per $10 \mu \mathrm{m}$ plasma membrane in 6-month-old male mice ( $n=3$ in each group). Scale bar: $50 \mathrm{~nm}$. All data are presented as mean \pm SEM. ${ }^{*} P<0.05,{ }^{* *} P<0.01,{ }^{* * *} P<0.001$.

knockdown of either Snap25 or Rab3d in MIN6 cells did not significantly alter GSIS (Figure 5E), a double knockdown of the exocytosis genes was necessary to significantly decrease GSIS and was consistent with the reconstitution data (Figure 5, D and E).

Consistent with the in vitro data, we observed a significant reduction of both $S n a p 25$ gene and Snap25 protein expression and an approximately 50\% reduction in Rab3d and Rab27a in islets isolated from RIP-TRB3F1 mice (Figure 5F). We also observed reduced expression of SNAP25 and RAB3D protein levels in human islets from T2DM patients compared with those of controls, and the reduction was more apparent in T2DM human islets isolated from a carrier with the Q84R polymorphism (Figure 5G). Consequently, electron microscopic analyses of the plasma membrane revealed a significantly reduced number of docked insulin granules under basal conditions in $\beta$ cells from the RIPTRB3F1 mice, without affecting the total granules per unit area (WT, $19.66 \pm 2.45$ granules per $\mu \mathrm{m}^{2}$; RIP-TRB3F1, $20.89 \pm 1.88$ granules per $\left.\mu \mathrm{m}^{2}\right)$ (Figure $5 \mathrm{H}$ ), and underscore the importance of docked granules for insulin exocytosis (23-25).

$84 R$ TRB 3 exhibits enhanced binding affinity to Akt and greater stability compared with Q84 TRB3. To dissect the molecular mechanism(s) underlying the differential phenotypic effects of Q84 versus 84R variants, we performed coimmunoprecipitation and stability experiments. Endogenous Trb3 and Akt proteins could be consistently coimmunoprecipitated in MIN6 cells, suggesting a basal level of interaction (Figure 6A). In addition, the 84R TRB3 protein coimmunoprecipitated with Akt at significantly elevated levels, compared with that of Q84 TRB3 protein, in the presence of equal amounts of total TRB3 (Q84 or 84R) and Akt (Figure 6B), suggesting enhanced binding affinity of 84R TRB3 for Akt. In studies on protein stability, we did not detect differences in expression of Q84 TRB3 or 84R TRB3 for up to 2 days after adenovirus infection (Figure 6C). However, on day 3, the level of 84R TRB3 was higher compared with that of Q84 TRB3, and the trend persisted until day 6 . Addition of the proteasomal inhibitor, MG132, virtu- ally completely prevented the degradation of Q84 TRB3 on day 4, while significant levels of Q84 TRB3 protein were preserved even up to day 6 , indicating that $84 \mathrm{R}$ TRB3 is relatively resistant to degradation. Concomitantly, the 84R TRB3 polymorphism also exhibited a greater inhibition of Akt phosphorylation (Figure 6C). Pulse-chase analysis to determine the half-life of TRB3 protein revealed a prolonged half-life (approximately 11 hours) for newly synthesized 84R TRB3 compared with the Q84 variant (Figure $6 \mathrm{D})$. Together, these data indicate that TRB3 is regulated by proteasomal degradation and that $84 \mathrm{R}$ TRB3 is more stable compared with Q84 TRB3.

Binding of TRB3 to ATF4 competitively inbibits binding of CRE with $C R E B$ on the promoters of exocytosis genes. Using a promoter analysis program, we identified CREB as a candidate transcription factor that mediates the effects of TRB3. Indeed, expressing constitutively active CREB (CA-CREB) alone increased the expression of Snap25 and Rab3d in MIN6 cells, while conversely, dominant-negative CREB (DN-CREB) reduced their expression (Figure 7A), suggesting that CREB links TRB3 to proteins involved in insulin exocytosis. Both Q84 and 84R TRB3 inhibited expression of Snap25 and Rab3d, which was successfully reversed when CA-CREB was coexpressed with either form of TRB3, and the inhibition was further enhanced when DN-CREB was coexpressed with either Q84 TRB3 or 84R TRB3 (Figure 7A). To confirm that CREB is involved in the regulation of exocytosis genes in silico, we identified CREB response elements on the Snap 25 promoter. Using ChIP assays, we observed that the CREB binding on Snap 25 promoter under basal conditions was enhanced when CREB was activated with forskolin. On the other hand, the ER stress inducer, thapsigargin, inhibited binding of CREB to the Snap25 promoter (Figure 7B).

Although neither polymorphic form of TRB3 physically interacted with CA-CREB or DN-CREB (data not shown), we observed that $84 \mathrm{R}$ TRB3 pulled down significantly more activating transcription factor 4 (ATF4, also known as CREB2) compared with Q84 TRB3 in coimmunoprecipitation assays (Figure 7C). Using reporter assays, we could demonstrate that Snap25 promoter activity was enhanced by CA-CREB and inhibited by DN-CREB overexpression (Figure 7D and Supplemental Figure 9). Expression of ATF4 also activated the Snap25 promoter and the promoter of the positive control, even-skipped homeobox homolog 1 (EVX1) (containing cAMP response element [CRE]) (26), albeit, to a lesser extent compared with CA-CREB (Figure 7D and Supplemental Figure 9). Therefore, in cells expressing ATF4, the latter competes with CREB for CREB sites at the Snap25 promoter to reduce transcription of Snap25. Interestingly, coexpression of Q84 TRB3 or 84R TRB3 with both CA-CREB and ATF4 significantly inhibited Snap25 promoter activity, with the 84R TRB3 variant exhibiting a significantly stronger inhibitory effect (Figure 7D). Similar results were obtained with the EVX-Luc-positive control construct (Supplemental Figure 9). These results suggest that TRB3 inhibits CREB-mediated transcription activities of exocytosis genes through ATF4 and that 84R TRB3 is a stronger binding partner of ATF4 and inhibitor compared with Q84 TRB3.

To explore the pathophysiological role of ATF4 and TRB3 on the expression of exocytosis genes, we induced ER stress in MIN6 cells using chemical inducers. ER stress induction was confirmed by the upregulation of ER stress markers Chop, Bip, spliced Xbp1, and Atf4 (Figure 7E and Supplemental Figure 10). ER stress induction using thapsigargin significantly increased Trb3 expression, while Snap25, Rab3d, Rab27a, and Vamp2 (Figure 7E) were all significantly 
A

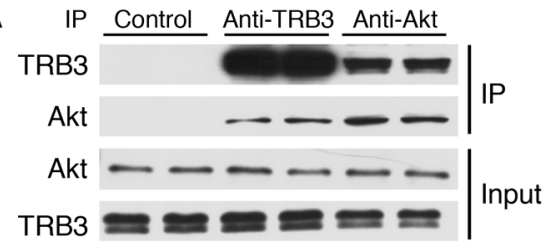

B IP:Akt Q84 84R

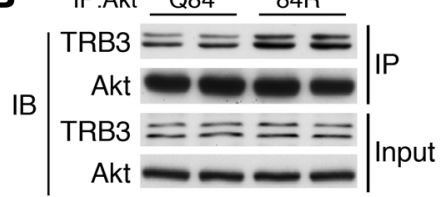

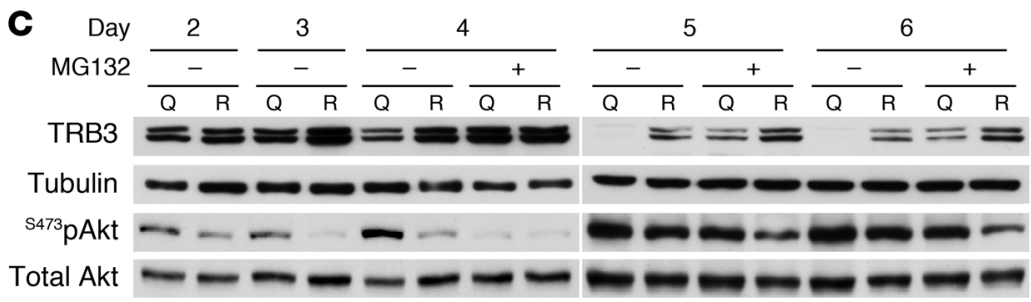

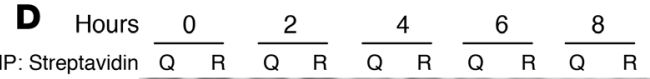

IB: TRB3 $\mathrm{a}=\mathrm{E}=\mathrm{z}=\mathrm{z}=$
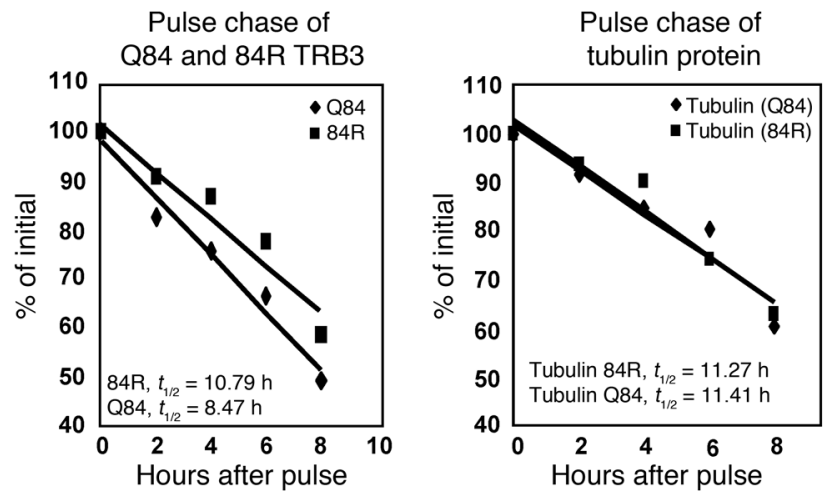

\section{Figure 6}

Enhanced TRB3 function in $\beta$ cells expressing 84R TRB3. (A) Immunoprecipitation for TRB3 and Akt in MIN6 cells. Cell lysate (input) and IP lysate (pull down) were analyzed by Western blotting. Representative images from 3 independent experiments are shown. (B) Immunoprecipitation for Akt and immunoblotting in MIN6 cells expressing either Q84 TRB3 or 84R TRB3. Cell lysate (input) and IP lysate (pull down) were analyzed by Western blotting. Representative images from 4 independent experiments are shown. (C) Western blotting for TRB3 and Akt in MIN6 cells expressing either Q84 TRB3 or 84R TRB3. Indicated samples were treated with the proteasome inhibitor MG132 (5 $\mu \mathrm{M}, 16$ hours prior to harvesting cells). Q, Q84 TRB3; R, 84R TRB3. Representative images from 3 independent experiments are shown. The white vertical line indicates noncontiguous lanes run on the same gel. (D) Pulse-chase experiment for TRB3 protein in MIN6 cells expressing either Q84 TRB3 or 84R TRB3 ( $n=3$ in each group). Q84 TRB3 or 84R TRB3 protein was labeled with modified methionine in vivo (pulse), then harvested at the indicated time points (chase), and immunoprecipitated with streptavidin beads. The rate of degradation of newly synthesized TRB3 and tubulin (internal control) was monitored by Western blotting, and the data was quantified and plotted as a percentage of initial labeled protein. downregulated. However, the expression of syntaxin1A (Stx1a), Munc-18a (Stxbp1), granuphilin (Sytl4), Rab3a, and Capn10 or the known CREB transcriptional target in $\beta$ cells, IRS2, was unaffected (Supplemental Figure 10). These results suggest that upregulation of TRB3 and ATF4 by a pathophysiological state, such as ER stress, downregulates CREB transcriptional activity that leads to a subsequent decrease in the expression of a specific subset of genes that are important for insulin exocytosis.

\section{Discussion}

While the mechanism(s) that promotes $\beta$ cell dysfunction in humans with T2DM is still poorly understood, recent reports indicate the involvement of ER stress (18) and impaired insulin secretion caused by altered insulin granule exocytosis $(27,28)$. Indeed, TRB3, a pseudokinase, has been reported to be induced by ER stress $(19,29)$ and upregulated in several pathological conditions $(30,31)$. We report here that TRB3 expression levels are upregulated in human and mouse pancreatic islets in multiple pathophysiological conditions characterized by altered glycemia. An increase in TRB3 levels in $\beta$ cells inhibits insulin signaling and leads to defective insulin exocytosis. Moreover, the polymorphic $84 \mathrm{R}$ variant, which is associated with increased risk of T2DM and cardiovascular disease in humans, exhibited a greater inhibition of $\beta$ cell secretory function. To our knowledge, these findings provide novel insights into molecular mechanisms that directly link TRB3 polymorphisms to altered $\beta$ cell exocytosis in the development of T2DM.

A polymorphism (rs2295490/Q84R) in exon 2 of TRB3, which changes glutamine to arginine in codon 84 , has been recently reported to be associated with T2DM, insulin resistance, and atherosclerosis in humans $(8,9,32)$. In the present study, in 2 populations of people of mixed European descent (Krakow, Poland, and Boston, Massachusetts, USA), individuals carriers for the minor allele coding for arginine (84R) exhibited an increased risk of T2DM in comparison with homozygotes of the major allele coding for glutamine (QQ84). In another recent study, in an Italian population, the $84 \mathrm{R}$ variant also exhibited significant association with early-onset T2DM and relative insulin deficiency (9) (V. Trischitta and L. Frittitta, personal communication). Unfortunately, the Q84R polymorphism is not in linkage disequilibrium with any of the other known HapMap SNPs, and, therefore, its effect on T2DM risk could not be examined in the publicly available genome-wide association studies $(33,34)$.

In addition to being expressed in islets, the TRB3 gene is reported to be expressed in the liver in humans; however, its expression levels in adipose, skeletal muscle, and brain are either low or undetectable (35). We pursued functional studies in $\beta$ cells, based on our findings of reduced C-peptide levels in carriers of the polymorphism and on the report by Prudente et al. (9) of insulin deficiency, which is likely due to defects in islet secretory function from individuals 


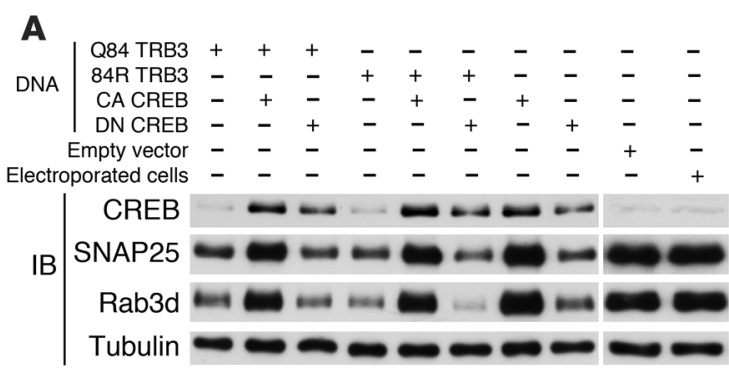

C

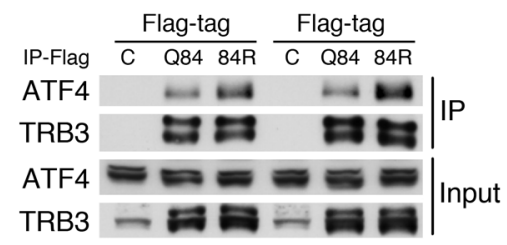

E

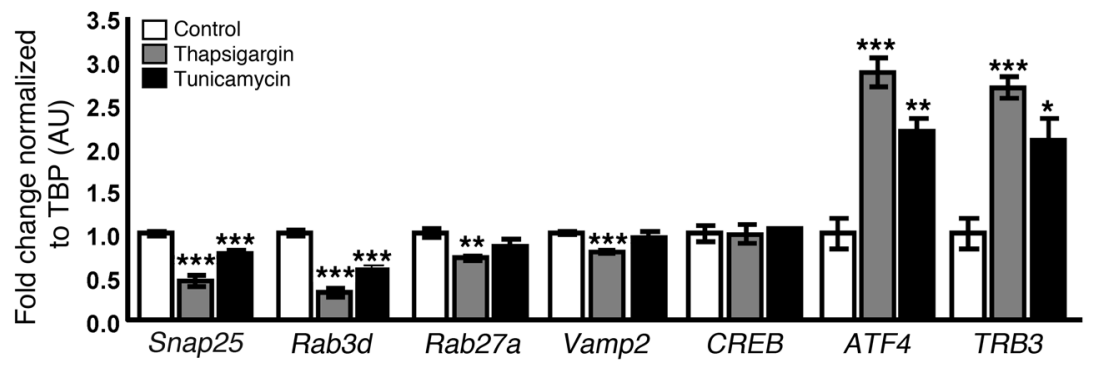

$\mathbf{F}$

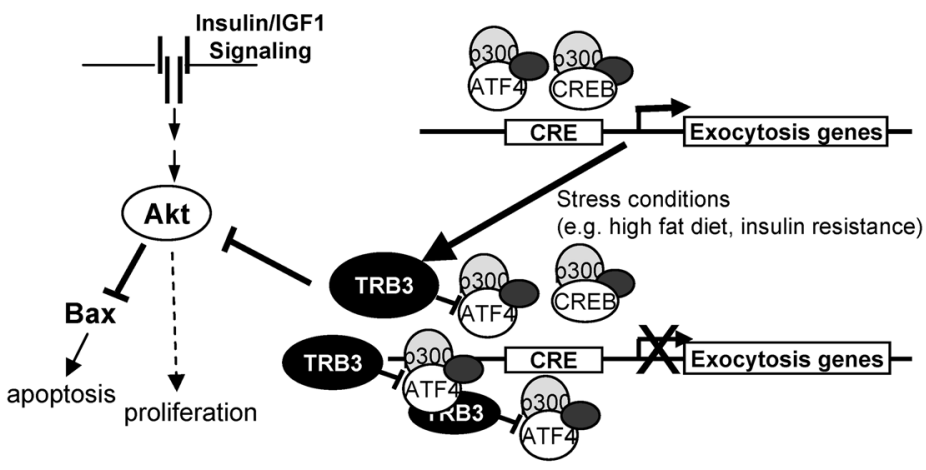

Figure 7

TRB3 and ATF4 inhibit expression of exocytosis genes. (A) Western blotting for CREB, SNAP25, and Rab3d in MIN6 cells electroporated with indicated plasmid DNAs. Representative images from 3 independent experiments are shown. The white vertical line indicates noncontiguous lanes. (B) ChIP assay for CREB on the Snap25 promoter in MIN6 cells treated with forskolin (10 nM, 1 hour) or thapsigargin (100 nM, 6 hours) ( $n=3$ in each group). The position of CREB-binding site relative to transcription start site (depicted by " +1 ") on Snap25 promoter is shown. (C) Immunoprecipitation for Flag-tag in MIN6 cells expressing either Flag-tag Q84 TRB3 or 84R TRB3. Cell lysate (input) and IP lysate (pull down) were analyzed by Western blotting. Representative images from 4 independent experiments are shown. (D) Luciferase reporter assay for the Snap25 promoter (SNAP25Pro) in HEK293 cells transiently transfected with plasmids as indicated ( $n=4$ in each group). Results were normalized to Renilla luciferase activity and expressed as relative luciferase units. ${ }^{* \star} P<0.001$ versus Snap25 promoter with CA-CREB. (E) qRT-PCR in MIN6 cells treated with either DMSO, thapsigargin (100 nM), or tunicamycin $(2 \mathrm{mg} / \mathrm{ml})$ for 8 hours $(n=3$ in each group). All data are presented as mean \pm SEM. ${ }^{\star} P<0.05,{ }^{\star *} P<0.01,{ }^{* \star *} P<0.001$. (F) Schematic depicting a potential role for TRB3 in the regulation of $\beta$ cell exocytosis, apoptosis, and proliferation. 
expressing the polymorphism in an independent cohort. Furthermore, the lack of specific phenotypic data in these individuals that directly point to functional defects in the liver also suggested that we should focus our studies on islets. Nevertheless, we plan to undertake functional studies in hepatocytes in a separate study.

Consistent with the reports that TRB3 expression levels are regulated by insulin signaling and stress $(19,29,36)$, we detected an increase in expression of TRB3 protein in islets from patients with $\mathrm{T} 2 \mathrm{DM}$, islets from obese and insulin resistant mouse models, and observed that insulin signaling negatively regulates TRB3 expression in pancreatic $\beta$ cells. These data, along with the observation that Q84R polymorphisms are associated with T2DM, implicate an important role for TRB3 in $\beta$ cell biology.

To study the role of TRB3 and the potential differential effect of the $84 \mathrm{R}$ polymorphism on islet $/ \beta$ cell function, we expressed the Q84 major variant or 84R minor variant of TRB3 in dispersed human islet cells or MIN6 cells to mimic the levels of TRB3 observed in diabetic islets (approximately 4-fold). Consistent with the effects observed in hepatocytes (7) and HUVECs, (37) both Q84 and 84R TRB3 blunted basal and insulin-stimulated Akt and ERK phosphorylation, with the latter variant having stronger inhibitory effects on Akt activation. Thus, the presence of a polymorphism (e.g., 84R) in TRB3 or a mere increase in expression of TRB3 can both induce an insulin resistant state and lead to poor proliferation and enhanced apoptosis of $\beta$ cells. Given that TRB3 expression is regulated by insulin signaling, a balance between insulin mediated-suppression of TRB3 and TRB3-mediated suppression of Akt might play a critical role in the regulation of $\beta$ cell function.

Expression of the TRB3 variants in $\beta$ cells resulted in a marked inhibition of glucose and non-glucose secretagogue-stimulated insulin secretion. In addition, $\beta$ cell-specific TRB3 overexpressing mice displayed impaired insulin secretion both in vivo and ex vivo. These data are consistent with significantly lower C-peptide levels in homozygous carriers of the 84R variant in humans in our studies and are in agreement with the relative insulin deficiency during an OGTT in humans carrying the 84R variant reported by Prudente et al. (10) and V. Trischitta and L. Frittitta (personal communication). Although the defects in GSIS observed in vivo in RIP-TRB3F1 mice occur when first-phase insulin secretion is expected, it would be desirable to confirm the defect using an islet perfusion model.

An alternative mechanism that determines the greater inhibitory effects of 84R TRB3 compared with Q84 TRB3 is the relatively greater stability of 84R TRB3, which potentially increased TRB3 expression and, together with the enhanced affinity of 84R TRB3 with Akt and ATF4, was able to alter islet secretory function and $\beta$ cell survival. The functional variation between Q84 and 84R TRB3 could also be due to differences in their conformational structures secondary to substitution of uncharged glutamine with charged arginine (38).

Consistent with a dominant effect of the $84 \mathrm{R}$ variant, the expression of Snap25 and Rab3d was inhibited in 84R TRB3-expressing $\beta$ cells and RIP-TRB3 mouse islets. Electron microscopic analysis of the plasma membrane of $\beta$ cells from the transgenic mice localized the site of defect to a significant decrease in the number of docked insulin granules. In addition, we identified the transcription factor CREB as an indirect target of TRB3 that potentially mediates the downregulation of Snap25 and Rab3d expression. We also observed that CREB binding on the Snap25 promoter is regulated by CREB activation and ER stress. ATF4, a member of the CREB family of transcription factors, consistently coimmunoprecipitated with TRB3 in $\beta$ cells, and the binding of TRB3 to ATF4 inhibited ATF4 transcription activity, providing evidence, for the first time to our knowledge, that ATF4 participates in the regulation of exocytosis in $\beta$ cells. Indeed, we observed that ATF4 competitively inhibited CREB activation of the Snap25 promoter. These data are supported by our observation that upregulation of TRB3 and ATF4 by ER stress induction downregulates Snap25 and point to ATF4 as a key player in the TRB3-CREB regulation of insulin exocytosis. The reversal in insulin secretion and expression of exocytosis genes in a loss-of-function model (knockdown) strengthens the argument for a role for TRB3 in $\beta$ cell exocytosis. Further studies are warranted to delineate the molecular pathway(s) and identify the proteins that link the TRB3 variants with Akt and modulate mitochondrial Bax to promote apoptosis in $\beta$ cells.

We propose that under normal conditions CREB regulates a subset of exocytosis genes by acting via CRE. However, in pathological states, such as obesity and diabetes, and/or when there is an increased demand for protein synthesis, the ER stress/unfolded protein response upregulates ATF4 and its downstream target, TRB3. Subsequently, TRB3 binds to ATF4 and promotes access to CRE sites, resulting in reduced SNAP25 promoter activity and a decrease in insulin exocytosis. Our findings demonstrate what we believe to be a novel regulation of a subset of exocytosis genes, whereby a transcriptionally inactive TRB3/ATF4 complex competitively inhibits CREB binding to CRE-binding sites, leading to downregulation of SNAP25 (Figure 7F).

In summary, the inhibitory role of TRB 3 in $\beta$ cell function, via impairment of insulin exocytosis and proliferation, and enhancement of apoptosis indicates the significance of targeting the tribbles protein for the development of new therapeutic approaches to prevent progressive $\beta$ cell dysfunction and improve $\beta$ cell survival in T2DM.

\section{Methods}

Genetic studies. Study protocols were approved by the Ethical Committee of the Medical School of the Jagiellonian University, Krakow, Poland, and the Joslin Diabetes Center. All participants signed informed consent forms at entry into the study. The clinical characteristics of the study groups are shown in Supplemental Table 2. The sequencing and genotyping methods are described in Supplemental Methods. The results of sequencing of all exons of TRB 3 and the distribution of the 6 identified sequence differences in patients from Krakow are shown in Supplemental Table 3. The distributions of the genotypes for Q84R polymorphism in exon 2 in the study groups are shown in Supplemental Table 1. (See Supplemental Methods for details.)

Animals and physiological studies. A 420-bp fragment of the RIP (RIP1) (39) and a $1-\mathrm{Kb}$ fragment encoding mouse TRB $3 \mathrm{cDNA}$ were subcloned into the pWhere vector containing $2 \mathrm{H} 19$ chromatin insulators. RIP-TRB3 transgenic mice were generated on the C57BL/6 background, and 2 founders, F1 and F2, were characterized. Mice were genotyped using the following primer sets: forward 1 RIP, GAGCTACACCTCTGGCTGCT; forward WT, TCTGCCTACCTACCCCTCCT; reverse, GGTGTACTCTGTGCCTGTGG. Mice were housed in pathogen-free facilities and maintained on a 12-hourlight/dark cycle at the Foster Biomedical Research Laboratory of Brandeis University in Waltham, Massachusetts, USA. All protocols were approved by the IACUC of the Joslin Diabetes Center and Brandeis University and were in accordance with NIH guidelines. Blood glucose, plasma insulin, glucose and insulin tolerance tests, and GSIS tests were performed as described previously (11). (See Supplemental Methods for details.)

Human islets. All human islets were obtained from the Southern California Islet Resources Center. Upon receipt, all human islets were cultured overnight in Miami Media \#1A (Cellgro) before being subjected to experimental proce- 
dures as described below. For insulin stimulation experiments, dispersed control human islet cells were washed with PBS and incubated in Functionality media (Cellgro) with $1 \%$ human serum albumin and $5.5 \mathrm{mM}$ glucose.

Antibodies. Rabbit polyclonal anti-TRB3 was raised against the peptide with the amino acid sequence TRB3-27-40, TERPVQKRARSGPQ, and affinity purified with TRB3-27-40 (BioGenes GmbH). Rabbit polyclonal anti-Akt, anti-phospho Akt (S473), anti-phospho Akt (T308), anti-p44/42 MAP kinase, anti-phospho-p44/42 MAP kinase, anti-AMPK $\alpha 1$, anti-phospho-AMPK $\alpha 1$ (S485), anti-cleaved caspase 3, and anti-CREB antibodies were purchased from Cell Signaling Technology; rabbit polyclonal anti-Bax, anti-HNF $4 \alpha$, anti-HNF1 $\alpha$, anti-Glut2, anti-GCK, anti-IR $\beta$, anti-IGF1R $\beta$, anti-Rab27a, and anti-ATF4 as well as goat polyclonal anti-Kir6.2 and antiSUR1 were obtained from Santa Cruz Biotechnology; rabbit polyclonal anti-Pdx1 was from Chemicon; rabbit polyclonal anti-IRS1 and anti-IRS2 were obtained from Upstate; mouse monoclonal anti-Nkx6.1 was obtained from Developmental Studies Hybridoma Bank; mouse monoclonal antiBrdU was obtained from Dako; and mouse monoclonal anti- $\alpha$-tubulin, anti-LaminB1, and anti-SOD4 and rabbit polyclonal anti-Rab3d, antiSNAP25, and anti-Vamp2 were purchased from Abcam.

Plasmids and siRNA. Full-length cDNA of TRB3 in PCMV6-XL5 vector was purchased from Origene Technology. Site-directed mutagenesis was performed with PCR, using oligos from Integrated DNA Technologies (IDT). Complete ORFs of SNAP25, Rab3d, and ATF4 as well as the SNAP25 promoter were amplified from MIN6 cell cDNA with PCR, using oligos from IDT, and cloned into PCDNA3.1 or pGL3 luciferase vector. Plasmids that encode CA-CREB and DN-CREB were used $(39,40)$. The EVX-1 luciferase construct was obtained from Marc Montminy (26) (Salk Institute). siRNAs against SNAP25 and Rab3d were obtained from Dharmacon. DN-Akt and CA-Akt constructs were obtained from C. Ronald Kahn (Joslin Diabetes Center).

Recombinant adenovirus. Viruses described in this study were constructed using the AdEasy Adenoviral System (Stratagene). Full-length Q84 and 84R TRB3 cDNAs were subcloned from pCMV6-XL5 into the pShuttle vector. After amplification, viruses were purified with the Adeno- $X$ Virus Purification Kit (Clontech) and titered with the Adeno-X Rapid Titer Kit (Clontech). The adenovirus for TRB3 RNAi used was obtained from Marc Montminy (41) (Salk Institute).

Coimmunoprecipitation and Western blotting. Coimmunoprecipitation and Western blotting were performed as described (42). (See Supplemental Methods for details.)

Proteasome or PI3K inhibition and ER stress induction. MIN6 cells were infected with respective recombinant adenoviruses and incubated for 24 hours. Cells with or without $5 \mu \mathrm{M}$ of proteasome inhibitor (MG132) (Calbiochem) were harvested at the indicated times with RIPA buffer and subjected to Western blotting. MG132 was added 16 hours prior to harvesting cells.

For PI3K inhibition, cells were pretreated with DMSO, $50 \mu \mathrm{M}$ of LY294002, or $25 \mu \mathrm{M}$ of wortmannin (Calbiochem) 4 hours before Western blotting. For ER stress induction, cells were treated with DMSO, $100 \mathrm{nM}$ of thapsigargin, or $2 \mu \mathrm{g} / \mathrm{ml}$ of tunicamycin (Calbiochem) for 8 hours before total RNA extraction.

Nonradioactive pulse chase. The Click-IT AHA (L-azidohomoalaine) for the nascent protein synthesis kit (Invitrogen) was used for these studies, and experiments were performed according to the manufacturer's recommendation. (See Supplemental Methods for details.)

Insulin secretion and insulin content. Human islets that were dispersed as previously described (43), islets that were obtained by collagenase digestion, or MIN6 cells were infected with respective recombinant adenovirus and incubated for 24 hours. Insulin secretion experiments were performed essentially as described (42). (See Supplemental Methods for details.)

Immunostaining and electron microscopy. Mice were anesthetized, and pancreata were rapidly dissected, weighed, processed, and immunostained as described previously (11). For electron microscopy, the pancreas was minced in fixative solution of $2.5 \%$ glutaraldehyde in a $0.1 \mathrm{M}$ phosphate buffer. Hand-dissected islets were post-fixed with a $2 \%$ osmium tetroxide solution in $0.1 \mathrm{M}$ phosphate buffer, infiltrated, and embedded with Araldite epoxy resin. Sections of $800 \AA \AA$ thickness were prepared from the islets. The thin sections were placed on copper mesh grids, stained with uranyl acetate and lead citrate before being observed, and photographed on the Philips 301 Transmission Electron Microscope. Docked granules were counted as described previously (24). (See Supplemental Methods for details.)

Luciferase assays. Cells were cotransfected with indicated firefly luciferase constructs and Renilla luciferase normalization control with Lipofectamine 2000. Lysates were collected 36 hours after transfection, and firefly and Renilla luciferase activities were measured with a Dual-Luciferase Reporter System (Promega).

ChIP. MIN6 cells under basal condition, treated with $100 \mathrm{nM}$ thapsigargin for 6 hours or $10 \mu \mathrm{M}$ forskolin for 1 hour, were prepared with the SimpleChIP Enzymatic Chromatin IP Kit (Cell Signaling Technology) according the manufacturer's protocol. In each case, the chromatin fractions were incubated with $10 \mu \mathrm{g}$ of one of the following antibodies at $4^{\circ} \mathrm{C}$ overnight: anti-CREB (Cell Signaling Technology) or normal Rabbit IgG (provided with SimpleChIP Kit, Cell Signaling Technology). Data are normalized by input values, and binding is expressed relative to the nonspecific binding of IgG immunoprecipitated DNA content. (See Supplemental Methods for details.)

Quantitative RT-PCR analysis. Quantitative RT-PCR analysis was performed as described previously (42). (See Supplemental Methods for details.)

Statistics. All data are presented as mean \pm SEM and analyzed using unpaired, 2-tailed Student's $t$ test or ANOVA, as appropriate. A $P$ value of less than 0.05 is considered significant.

\section{Acknowledgments}

The authors thank M. Montminy and C.R. Kahn for providing reagents and discussions; J. Pessin, G.W. Sharp, and S. BonnerWeir for advice; L. Huse and E. Morgan for excellent assistance in the preparation of this manuscript; $\mathrm{H}$. Li for assistance with hormone assays; and C. Cahill for assistance with electron microscopy. This work was supported by NIH grant RO1 DK 67536 and the Graetz Bridge Funds (to R.N. Kulkarni), NIH grant RO1 DK47475 (to A.S. Krolewski), NIH grant RO1 TW01351 (to A.S. Krolewski), and the Joslin DERC Specialized Assay and Advanced Microscopy Cores (NIH P30 DK36836). C.W. Liew was supported by a Mary K. Iacocca Postdoctoral Fellowship and an NIH Interdisciplinary training grant (1RL9EB008539-01) (SysCODE). D. Kawamori is the recipient of a research fellowship (Manpei Suzuki Diabetes Foundation, Japan) and a JDRF postdoctoral fellowship.

Received for publication February 10, 2010, and accepted in revised form May 19, 2010.

Address correspondence to: Rohit N. Kulkarni, Joslin Diabetes Center, Room 602, One Joslin Place, Boston, Massachusetts 02215, USA. Phone: 617.713.3460; Fax: 617.713.3476; E-mail: Rohit. Kulkarni@joslin.harvard.edu.
1. Wild S, Roglic G, Green A, Sicree R, King H. Global prevalence of diabetes: estimates for the year 2000 and projections for 2030. Diabetes Care. 2004;27(5):1047-1053.
2. Reaven GM. Banting lecture 1988. Role of insulin resistance in human disease. Diabetes. 1988; 37(12):1595-1607.

3. Kahn CR. Banting Lecture. Insulin action, diabe- togenes, and the cause of type II diabetes. Diabetes. 1994;43(8):1066-1084.

4. Accili D. Lilly lecture 2003: the struggle for mastery in insulin action: from triumvirate to republic. Dia- 
betes. 2004;53(7):1633-1642.

5. Elghazi L, Bernal-Mizrachi E. Akt and PTEN: betacell mass and pancreas plasticity. Trends Endocrinol Metab. 2009;20(5):243-251.

6. Taniguchi CM, Emanuelli B, Kahn CR. Critical nodes in signalling pathways: insights into insulin action. Nat Rev Mol Cell Biol. 2006;7(2):85-96.

7. Du K, Herzig S, Kulkarni RN, Montminy M. TRB3: a tribbles homolog that inhibits Akt/PKB activation by insulin in liver. Science. 2003;300(5625):1574-1577.

8. Prudente S, et al. The functional Q84R polymorphism of mammalian Tribbles homolog TRB3 is associated with insulin resistance and related cardiovascular risk in Caucasians from Italy. Diabetes. 2005;54(9):2807-2811.

9. Prudente S, et al. The TRIB3 Q84R polymorphism and risk of early-onset type 2 diabetes. J Clin Endocrinol Metab. 2009;94(1):190-196.

10. Otani K, et al. Reduced beta-cell mass and altered glucose sensing impair insulin-secretory function in betaIRKO mice. Am J Physiol Endocrinol Metab. 2004;286(1):E41-E49.

11. Kulkarni RN, Brüning JC, Winnay JN, Postic C, Magnuson MA, Kahn CR. Tissue-specific knockout of the insulin receptor in pancreatic beta cells creates an insulin secretory defect similar to that in type 2 diabetes. Cell. 1999;96(3):329-339.

12. Mathis D, Vence L, Benoist C. beta-Cell death during progression to diabetes. Nature. 2001; 414(6865):792-798.

13. Kim SJ, Winter K, Nian C, Tsuneoka M, Koda Y, McIntosh CH. Glucose-dependent insulinotropic polypeptide (GIP) stimulation of pancreatic betacell survival is dependent upon phosphatidylinositol 3-kinase (PI3K)/protein kinase B (PKB) signaling, inactivation of the forkhead transcription factor Foxo1, and down-regulation of bax expression. J Biol Chem. 2005;280(23):22297-22307.

14. Kiss-Toth E, et al. Human tribbles, a protein family controlling mitogen-activated protein kinase cascades. J Biol Chem. 2004;279(41):42703-42708.

15. Fu A, et al. Loss of Lkb1 in adult beta cells increases beta cell mass and enhances glucose tolerance in mice. Cell Metab. 2009;10(4):285-295.

16. Koh HJ, et al. Skeletal muscle-selective knockout of LKB1 increases insulin sensitivity, improves glucose homeostasis, and decreases TRB3. Mol Cell
Biol. 2006;26(22):8217-8227.

17. Qi L, et al. TRB3 links the E3 ubiquitin ligase COP1 to lipid metabolism. Science. 2006; 312(5781):1763-1766.

18. Fonseca SG, Lipson KL, Urano F. Endoplasmic reticulum stress signaling in pancreatic beta-cells. Antioxid Redox Signal. 2007;9(12):2335-2344.

19. Ohoka N, Yoshii S, Hattori T, Onozaki K, Hayashi H. TRB3, a novel ER stress-inducible gene, is induced via ATF4-CHOP pathway and is involved in cell death. EMBO J. 2005;24(6):1243-1255.

20. Aizawa T, Komatsu M. Rab27a: a new face in beta cell metabolism-secretion coupling. J Clin Invest. 2005;115(2):227-230.

21. Iezzi M, et al. Subcellular distribution and function of Rab3A, B, C, and D isoforms in insulin-secreting cells. Mol Endocrinol. 1999;13(2):202-212.

22. Sreenan SK, et al. Calpains play a role in insulin secretion and action. Diabetes. 2001;50(9):2013-2020.

23. Ohara-Imaizumi M, Cardozo AK, Kikuta T, Eizirik DL, Nagamatsu S. The cytokine interleukin1 beta reduces the docking and fusion of insulin granules in pancreatic beta-cells, preferentially decreasing the first phase of exocytosis. J Biol Chem. 2004;279(40):41271-41274.

24. Straub SG, Shanmugam G, Sharp GW. Stimulation of insulin release by glucose is associated with an increase in the number of docked granules in the beta-cells of rat pancreatic islets. Diabetes. 2004; 53(12):3179-3183.

25. Rorsman $\mathrm{P}$, Renstrom E. Insulin granule dynamics in pancreatic beta cells. Diabetologia. 2003; 46(8):1029-1045

26. Conkright MD, et al. TORCs: transducers of regulated CREB activity. Mol Cell. 2003;12(2):413-423.

27. Michael DJ, Cai H, Xiong W, Ouyang J, Chow RH. Mechanisms of peptide hormone secretion. Trends Endocrinol Metab. 2006;17(10):408-415

28. Ostenson CG, Gaisano H, Sheu L, Tibell A, Bartfai $\mathrm{T}$. Impaired gene and protein expression of exocytotic soluble $\mathrm{N}$-ethylmaleimide attachment protein receptor complex proteins in pancreatic islets of type 2 diabetic patients. Diabetes. 2006;55(2):435-440

29. Ord D, Ord T. Characterization of human NIPK (TRB3, SKIP3) gene activation in stressful conditions. Biochem Biophys Res Commun. 2005;330(1):210-218.

30. Shang YY, et al. TRB3, upregulated by ox-LDL, mediates human monocyte-derived macrophage apoptosis. FEBS J. 2009;276(10):2752-2761.

31. Xu J, et al. TRB3 interacts with CtIP and is overexpressed in certain cancers. Biochim Biophys Acta. 2007;1770(2):273-278.

32. Gong HP, et al. TRIB3 functional Q84R polymorphism is a risk factor for metabolic syndrome and carotid atherosclerosis. Diabetes Care. 2009; 32(7):1311-1313.

33. Zeggini E, et al. Meta-analysis of genome-wide association data and large-scale replication identifies additional susceptibility loci for type 2 diabetes. Nat Genet. 2008;40(5):638-645.

34. Saxena R, et al. Genome-wide association analysis identifies loci for type 2 diabetes and triglyceride levels. Science. 2007;316(5829):1331-1336.

35. Su AI, et al. A gene atlas of the mouse and human protein-encoding transcriptomes. Proc Natl Acad Sci US A. 2004;101(16):6062-6067.

36. Ding J, Kato S, Du K. PI3K activates negative and positive signals to regulate TRB3 expression in hepatic cells. Exp Cell Res. 2008;314(7):1566-1574.

37. Andreozzi F, et al. TRIB3 R84 variant is associated with impaired insulin-mediated nitric oxide production in human endothelial cells. Arterioscler Thromb Vasc Biol. 2008;28(7):1355-1360.

38. De Cosmo S, et al. Glutamine to arginine substitution at amino acid 84 of mammalian tribbles homolog TRIB3 and CKD in whites with type 2 diabetes. Am J Kidney Dis. 2007;50(4):688-689.

39. Jhala US, et al. cAMP promotes pancreatic beta-cell survival via CREB-mediated induction of IRS2. Genes Dev. 2003;17(13):1575-1580.

40. Asahara $\mathrm{H}$, et al. Chromatin-dependent cooperativity between constitutive and inducible activation domains in CREB. Mol Cell Biol. 2001; 21(23):7892-7900.

41. Koo SH, et al. PGC-1 promotes insulin resistance in liver through PPAR-alpha-dependent induction of TRB-3. Nat Med. 2004;10(5):530-534.

42. Borowiec M, et al. Mutations at the BLK locus linked to maturity onset diabetes of the young and \{beta\}-cell dysfunction. Proc Natl Acad SciUS A. 2009;106(34):14460-14465

43. Josefsen K, et al. Fluorescence-activated cell sorted rat islet cells and studies of the insulin secretory process. J Endocrinol. 1996;149(1):145-154. 